\title{
Utilization of ActiGraft, an Autologous (Blood Clot) Graft in the Reconstruction of Soft Tissue Deficit from Hand Trauma - A Case Study
}

\author{
Richard D Curtis MD ${ }^{1}$ and Chinenye D Wachuku DPM ${ }^{2 *}$ \\ ${ }^{1}$ Hand \& Wrist Center, LLC, Fleming Island, Florida \\ ${ }^{2}$ Barry University School of Podiatric Medicine, Florida
}

Submission: April 29, 2021; Published: May 07, 2021

"Corresponding author: Chinenye D Wachuku, Barry University School of Podiatric Medicine, Miami, Florida

\begin{abstract}
The reconstruction of complex soft tissue deficits is associated with great difficulty. When treating intricate structures of the hand, optimal reconstruction is necessary to preserve the function and aesthetics of the hand, preferably to the level associated with preinjury. The mechanism of scar formation is a process that is targeted by many researchers in an attempt to lessen both clinical and financial burden. Excessive hypertrophic tissue can cause adverse events, such as obstruction of vital structures, tendons and nerves, which can reduce the overall function of the hand and the patient's quality of life. ActiGraft, an autologous blood clot, provides effective wound healing and reconstruction of soft tissue, whilst mitigating hypertrophic scar tissue. ActiGraft expedited the process of revitalization of the wound by promoting ingrowth of healthy granulation tissue and reduction in excessive scar tissue formation. Here we present a case of ActiGraft treatment utilized weekly to promote soft tissue reconstruction of the hand, as well as modification of the mechanism required for scar tissue accumulation. Blood was withdrawn from the patient, at the pointof-care, to create a blood clot that was applied to the wound area. This treatment exhibited significant promotion of granulation tissue ingrowth, repairing the injured soft tissue and skin deficit. ActiGraft was found to be a cost-effective treatment that provided optimal and effective healing of the deficit related to the trauma of the hand.
\end{abstract}

Keyword: Autologous; Blood clot; Soft tissue; Reconstruction; Graft; Scar; Wound; Skin substitute

Abbreviations: ECM: Extracellular Matrix; ITT: Intent to Treat; PP: Per Protocol; PAR: Percentage Area Reduction

\section{Introduction}

Reconstruction of the soft tissue envelope of the hand is a difficult task to accomplish due to the ability to preserve the intricate elements embedded in the hands necessary for tasks such as gross and fine motor activity, tactile function, and perception capabilities. Severe damage to the hands can occur easily as a result of lack of abundant skin, fat, and muscle to the area, which would protect neurovascular and musculotendinous structures. When reconstructing skin deficits of the hand, one must be aware of the process associated with scar formation. An increase in scar formation can affect the wound healing process negatively by causing tension to the area, limiting and delaying adequate closure of the wound bed. Scar formation is a process that is still difficult to treat and prevent despite many attempts to mitigate the progression of scars. Addressing the potential for scar formation to take place has been a target of many therapies. Cutaneous wound healing following injury is a complex biological process that involves the collaboration of different immunological mediators, inflammatory pathways, mechano transduction pathways, and various cells [1]. During wound healing, towards the end of the proliferative phase into the remodeling phase, tissue deficits are replenished with different cells, mainly fibroblasts and a disorganized collagen-rich extracellular matrix. This combination results in a scar. Cutaneous scarring causes substantial psychological and physiological strain on patients. It is estimated that approximately $\$ 12$ billion is spent annually on scar treatments in the U.S. alone. [1]. Over 40 million patients undergo surgical procedures annually, increasing the number of patients, as well as the economic impact associated with wound and scar treatment [1].

Currently, the gold standard of treatment for diminishing the presence of scars are the use of corticosteroid injections, cryotherapy, surgical revisions, topical silicone gels, radiation, laser treatments, etc. These therapies have exhibited mixed results, obtaining some success, partial alleviation, and failures. 
An ideal treatment would be autologous, without the need for a split or full thickness graft, to avoid donor site morbidity [2]. A skin substitute must be utilized to not only consolidate the wound bed but to also mitigate scar formation, allowing for sufficient wound healing to occur. ActiGraft, an autologous blood clot, is a point-of-care treatment that functions as a skin substitute by utilizing the patient's own blood to create a matrix scaffold. The autologous matrix stimulates the expression of mediators necessary in regulating the inflammatory pathways, as well as factors necessary for temporary protection while the body generates a new extracellular matrix (ECM) and remodels the wound bed. ActiGraft was found to be safe and effective in clinical studies on cutaneous wounds $[3,4]$. We report here a case study of a cutaneous deep injury, treated with ActiGraft as a means of simultaneous wound healing and mitigation of scar formation.

\section{Case Presentation}

A 60-year-old male, with no past medical history, was admitted to the emergency room due to a gunshot wound to the left hand. A surgical washout operation was performed with a following repeated surgical washout, removal of debris, and nerve and muscle repair 2 days after. During this second procedure, salvageable skin was sutured via primary closure, and the patient was treated with antibiotics and local wound care. At this time, his wound exhibited a significantly deep soft tissue deficit measuring $8.2 \times 5.3 \mathrm{~cm}$. Due to the substantial loss of soft tissue structures of the hand, treatment options such as a skin flap could not be utilized. Assessment of the wound 10 days post-surgical treatment displayed some dehiscence noted to the surgical site and necrotic tissue was observed with no decrease in wound size. The patient reported experiencing pain in the wound area. The patient's sutures were removed, and sharp debridement was initiated to the wound area in order to remove the necrotic tissue. No improvement was observed during the one-week follow-up with no decrease in wound size, exhibited a wound bed "consisting of necrosis," slough, and fibrotic tissue. Advanced regenerative treatments were applied with no change to the wound condition. A significant deficit to the injured area of the hand was still present without improvement from the last visit.

ActiGraft treatment was applied to mitigate wound deterioration. After one application the wound decreased by $73 \%$ in size, demonstrating the ability of ActiGraft to initiate wound healing properties in deteriorating wounds. A weekly application took place, thereafter, showing a continued decrease in wound size and resolution in the depth of the skin deficit (Figure 1). The wound bed had improved with the eradication of the previously observed necrotic tissue, and a substantial reduction in fibrotic tissue and slough was noted. (Figure 2). On Week 5, the fourth and final ActiGraft treatment was applied to the wound. The injured area continued to coalesce, and the wound bed no longer exhibited any fibrotic or necrotic tissue, as well as no presence of slough. The wound bed consisted of healthy granulated tissue, representing proper wound healing (Figure 3). Complete wound healing and closure were achieved on week 10 post-surgical with minimal scar formation (Figure 4). Due to the mitigation of scar formation via ActiGraft application, there was less tension to the wound edges which prevented scar tissue from obstructing functionality of vital structures such as tendons and nerves regarding hand mobility, fine motor activity, tactile functionality, and capabilities of perception.

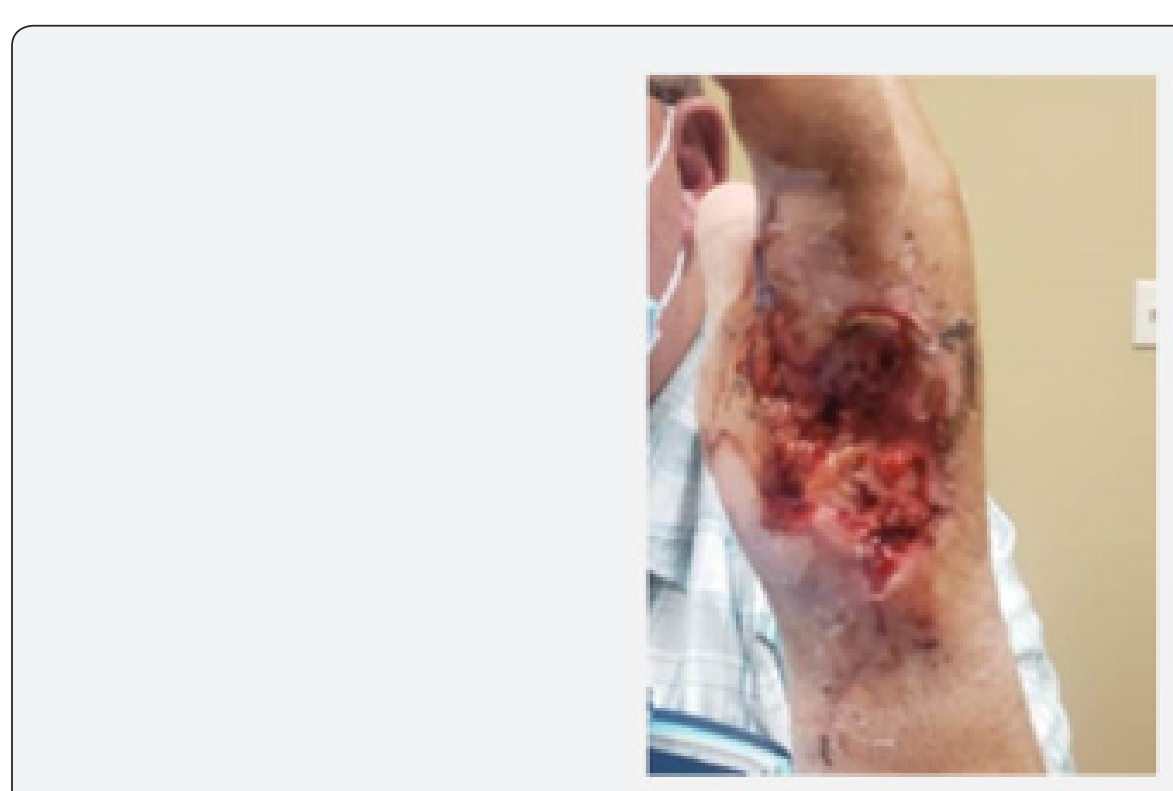

Figure 1: Patient wound at baseline, prior to ActiGraft's first application. The injured area exhibited mild erythema and edema surrounding the wound edges. The wound bed contained various area of necrosis, slough, as well as fibrotic tissue which is consistent with a non-healing wound. 


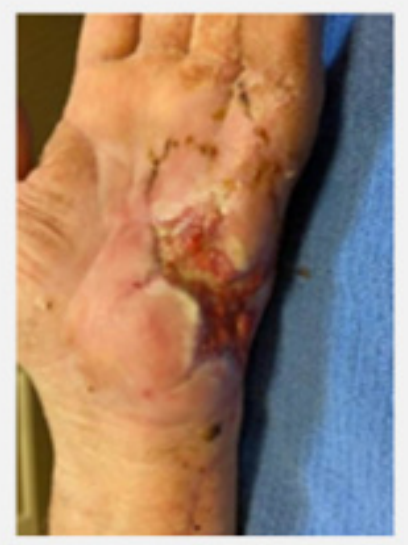

Figure 2: Substantial wound reduction following only one ActiGraft application. One week following the first Actigraft application, a significantly wound size reduction was observed with no signs of necrosis. There was a substantial decrease in the presence of slough and fibrotic tissue.

Figure 3: Increased granulation noted after the fourth application of the ActiGraft treatment. The wound bed showed signs of continued coalescence and no longer exhibited fibrotic tissue and slough. Healthy granulation tissue presents which is consistent with the presence of a properly healing wound.
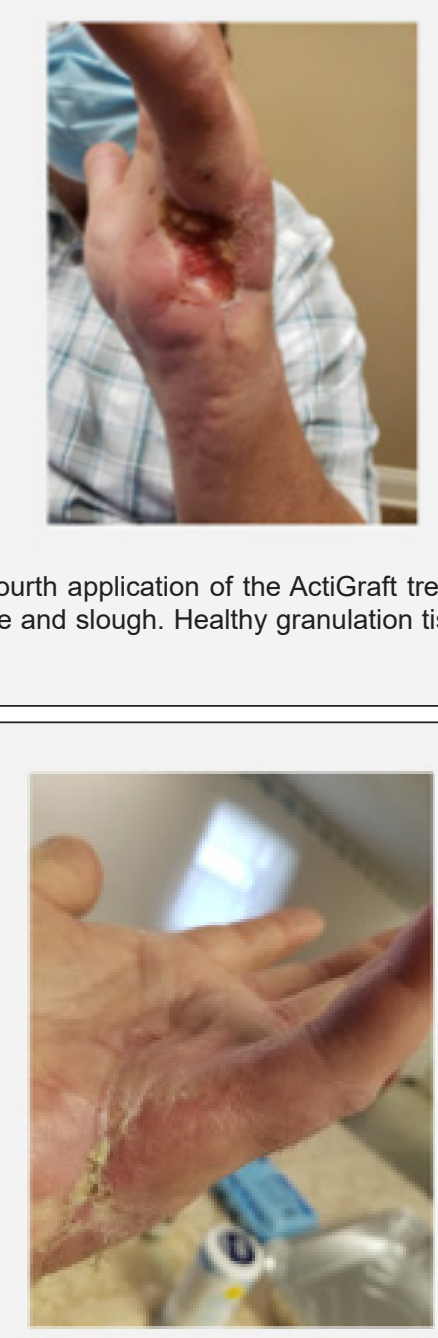

Figure 4: Complete closure of the wound bed was noted with a significant reduction in scar tissue after five ActiGraft applications. The injured area presented to be completely healed and there were no signs of infection. 


\section{Discussion}

Scar formation is a challenging concern that has brought about immense clinical, as well as a financial burden on the healthcare system. There is a great economic impact when the costs of disability and revision surgeries, due to dysfunctional tissue and disfiguring scars, are included [5]. Hypertrophic tissue formation occurs due to various factors ranging from inflammatory mishaps as well as disorganization of mechanisms of wound healing. When scars are produced excessively, it results in poor functional and aesthetic outcomes due to the formation of hypertrophic scar tissue and keloids [5]. Research has been done to target different aspects of the critical components of scar tissue development as a means of creating effective treatment options to mitigate the process.

Utilizing ActiGraft, an autologous skin graft, stimulates the patient's own wound healing mechanism to enhance wound healing and decrease fibrosis after wounding [6]. Consistent ActiGraft application altered the course of the wound, changing it from its deteriorating status to one that displays adequate progression through the wound healing process. In this case, "ActiGraft provided an environment suitable for sufficient wound healing, soft tissue reconstruction, and decreased scar formation. ActiGraft provided a moist environment that promotes epithelization and reduced scar formation assisting in decreasing" any tension that would normally arise due to hyperkeratotic or excessive scar tissue [7]. Reduction of tension is paramount in preserving soft tissue structures such as tendons and nerves that are responsible for maintaining the functionality of the hand. The autologous skin graft establishes an interim ECM not only as a means of protection, but also as a means of providing the necessary scaffold responsible for facilitating soft tissue reconstruction of the hand while also engaging cytokines, growth factors, and other mediators that are essential for soft tissue repair. The autologous characteristic of the skin graft aided in the organized replacement of the ECM which was damaged as a consequence of wounds or injury [7]. By decreasing the need for excess fibrosing of the tissues, ActiGraft not only successfully repaired the skin deficit, but also simultaneously mitigated scar formation while supporting good hand mobility [8-12].

Moreover, ActiGraft treatment was found to be cost-effective in comparison to alternative treatments. Whereas the modality of other treatments varies from $\$ 1,060$ to $\$ 4,840$ of 4 weeks of treatment, ActiGraft treatment lowers the cost by more than $50 \%$ "with a cost range of $\$ 1,000$ to $\$ 2,000$ for the equivalent time period.[8]. ActiGraft treatment showed high efficacy in complex wounds with a potential to dramatically reduce the financial cost on the health system.

\section{Conclusion}

ActiGraft prepares the skin deficit and provides necessary elements required to not only promote wound healing and reconstruction of soft tissue deficits, but to also curtail excessive scar formation. ActiGraft, a point-of-care treatment, integrates the patient's own blood as a means of formulating a matrix scaffold. The newly formed matrix scaffold then works to reconcile the wound bed, while simultaneously reducing excessive scar formation. ActiGraft was shown to be effective in promoting ingrowth of healthy granulation tissue and provides a moist environment with the organization of factors important for mitigating scar formation. A weekly application of ActiGraft decreased the wound bed size and depth and was effective in reducing hypertrophic tissue buildup. ActiGraft is a cost-effective measure that can accelerate the wound healing process efficiently while generating the functionality of vital structures resulting in this case with complete hand mobility.

\section{References}

1. Kwon SH, Padmanabhan J, Henn D, Chen K, Gurtner GC (2020) New Drugs for Scar Treatment. Textbook on Scar Management pp. 457-463.

2. Friedrich JB, Katolik LI, Vedder NB (2009) Soft Tissue Reconstruction of the Hand. The Journal of Hand Surgery 34(6): 1148-1155.

3. Snyder RJ, Schultz G, Wachuku C, Rashid AM, Ead JK (2020) Proposed Mechanism of Action of Topically Applied Autologous Blood Clot Tissue: A Quintessential Cellular and Tissue Based Therapy. J Am Podiatr Med Assoc pp. 20-140.

4. Kushnir I, Kushnir A, Garfinkel D, Serena TE (2016) Efficacy and Safety of a Novel Autologous Wound Matrix in the Management of Complicated, Chronic Wounds: A Pilot Study. Wounds Research 28(9): 317-327.

5. Barnes LA, Marshall CD, Leavitt T, Hu MS, Moore AL, et al. (2018) Mechanical Forces in Cutaneous Wound Healing: Emerging Therapies to Minimize Scar Formation. Advances in Wound Care 7(2): 47-56.

6. Yu J, Wang MY, Tai HC, Cheng NC (2018) Cell sheet composed of adiposederived stem cells demonstrates enhanced skin wound healing with reduced scar formation. Acta Biomaterialia 77: 191-200.

7. Ozker MDE, Wachuku, MEng C (2021) The Use of ActiGraft, an Autologous Skin Graft, in the Treatment of Complex Diabetes Foot Ulcer - A Case Study. Annals of Reviews and Research 6(2).

8. Snyder R, Ead JK (2020) A Comparative Analysis of the Cost Effectiveness of Five Advanced Skin Substitutes in the Treatment of Foot Ulcers in Patients with Diabetes. Annals of Reviews and Research 6(1).

9. Monavarian M, Kader S, Moeinzadeh S, Jabbari E (2019) Regenerative Scar-Free Skin Wound Healing. Tissue Engineering Part B: Reviews 25(4): 294-311.

10. Wu G, Ma X, Fan L, Gao Y, Deng H, Wang Y (2020) Accelerating dermal wound healing and mitigating excessive scar formation using LBL modified nanofibrous mats. Materials \& Design 185: 108265.

11. Willem M van der Veer, Bloemen MCT, Ulrich MMW, Grietje Molema, Paul P van Zuijlen, et al. (2009) Potential cellular and molecular causes of hypertrophic scar formation. Burns 35(1): 15-29.

12. Chen L, Wang J, Li S, Zhou Yu, Bei Liu, et al. (2018) The clinical dynamic changes of macrophage phenotype and function in different stages of human wound healing and hypertrophic scar formation. International Wound Journal 16(2): 360-369. 
This work is licensed under Creative Commons Attribution 4.0 License

DOI:10.19080/ARR.2021.06.555692
Your next submission with Juniper Publishers will reach you the below assets

- Quality Editorial service

- Swift Peer Review

- Reprints availability

- E-prints Service

- Manuscript Podcast for convenient understanding

- Global attainment for your research

- Manuscript accessibility in different formats

( Pdf, E-pub, Full Text, Audio)

- Unceasing customer service

Track the below URL for one-step submission https://juniperpublishers.com/online-submission.php 